\title{
Posterior Arthroscopic Subtalar Arthrodesis
}

\author{
Bernhard Devos Bevernage, MD, Laurent Goubau, MD, Paul-André Deleu, MSc, Vincent Gombault, MD, \\ Pierre Maldague, MD, and Thibaut Leemrijse, MD \\ Published outcomes of this procedure can be found at: Orthop Traumatol Surg Res. 2011 June;97(4):401-5
}

\section{Introduction}

Although subtalar joint arthrodesis may be achieved through open or arthroscopic approaches, we have found that posterior arthroscopic subtalar arthrodesis has technical advantages compared with other approaches (Video 1). There are several arthroscopic options, with the sinus tarsi approach traditionally seen as the most accessible to all subtalar facets ${ }^{1-3}$. However, in our experience, the posterior arthroscopic approach has shown more effective results that are probably due to better preservation of the talar vascularization $n^{4-9}$.

With the patient in the prone position and after the placement of both posterior portals around the ankle, the posterior facet of the subtalar joint can be reached and entered carefully from posterior to anterior, so that the joint can be debrided and microfractured until bleeding bone appears ${ }^{4}$ (Video 2). The fixation of the joint can be done afterward by using cannulated screws to achieve a stable construction enabling ossification between talus and calcaneus ${ }^{4}$.

\section{Step 1: Preoperative Assessment and} Planning (Video 3)

Clinically assess the foot and ankle with regard to remaining motion, previous scarring, and associated deformities, ensuring that no concomitant procedure will be needed in the supine position.

- Look for signs of posterior impingement syndrome, such as tendinitis of the flexor hallucis longus in the posteromedial region of the ankle; a painful posterior impingement test, with concomitant osseous avulsions; a hypertrophic posterior process of the talus; and os trigonum.

- Always use standard radiographs with a hindfoot alignment view, such as the Saltzman ${ }^{10}$ or the Méary ${ }^{11}$ view. Use computed tomography (CT) scanning, magnetic resonance imaging (MRI), or single-photon emission CT (SPECT) to evaluate the amount of bone loss, to assess associated degenerative changes in adjacent joints, and to look for other pathologies in the posterior compartment of the ankle.
- The possibility of obtaining good results with the posterior arthroscopic subtalar arthrodesis (PASTA) procedure depends on the decision algorithm shown in Figure 1.

\section{Step 2: Positioning of the Patient (Video 4)}

Use spinal or general anesthesia.

- Use a single preoperative dose of prophylactic antibiotics intravenously before inflating the tourniquet, which is applied around the thigh.

- Place the patient in the prone position on a radiolucent table.

- Place a support against the ipsilateral buttock to enable tilting of the table so that the ankle is straight and perpendicular to the floor.

- Place a support under the foot to allow the ankle to move freely in varus, valgus, flexion, and extension.

- Prepare and drape the limb distal to the knee.

- Exsanguinate the limb prior to inflating the tourniquet.

Step 3: Placement of the Posterolateral

Portal Lateral to the Achilles Tendon

(Fig. 2, Video 4)

Place the posterolateral portal lateral to the Achilles tendon at the level of the tip of the lateral malleolus, according to the original technique described by van Dijk et al. ${ }^{12}$.

- First, mark the tip of the lateral malleolus.

- Mark the lateral border of the Achilles tendon.

- Draw a line between those two landmarks, while holding the ankle in neutral position.

- Make a vertical stab incision on the lateral border of the Achilles tendon, proximal to this line.

- Direct a closed mosquito clamp anteriorly, pointing in the direction of the interdigital web space of the first and second toes. 
- When touching the bone, try to feel the curvature of the posterior aspect of the talus and open the mosquito clamp while extracting it. Exchange the mosquito clamp for a 4.5-mm arthroscope shaft with a blunt trocar pointing in the same direction.

- Distinguish the ankle joint from the subtalar joint. Therefore, find the posterior prominence of the posterior talar process lying in between both joints.

Step 4: Placement of the Posteromedial Portal Medial to the Achilles Tendon

(Fig. 3, Video 4)

Place the posteromedial portal medial to the Achilles tendon at the level of the tip of the lateral malleolus ${ }^{12}$.

- Extend the line drawn earlier further medially, crossing the Achilles tendon.

- Make the posteromedial portal just medial to the Achilles tendon at the same level of the posterolateral portal in the coronal plane.

- After the skin incision is made, introduce a mosquito clamp and direct it toward the arthroscope shaft.

- Use the shaft as a guide to travel anteriorly in the direction of the ankle joint and ensure that the mosquito clamp touches the arthroscope all of the way until it reaches the bone. In this way, the neurovascular bundle is avoided.

- Exchange the blunt trocar for a 4.0-mm $30^{\circ}$ arthroscope.

- The direction of view is to the lateral side to prevent damage to the lens system.

- Pull the arthroscope slightly backward and tilt it, until the tip of the mosquito clamp comes into view. Use gravity flow.

- Use the clamp to spread the extra-articular soft tissue just in front of the tip of the arthroscope.

- Exchange the mosquito clamp for a 5-mm fullradius shaver.

Step 5: Exposure of the Subtalar Joint by Removing the Fatty Tissue and Part of the Posterior Joint Capsule

(Fig. 4, Video 5)

With the help of the shaver, look for the landmarks of the posterior arthroscopy, going from the posterolateral part of the subtalar joint toward the flexor hallucis longus tendon, which is medially located, while exposing the tibiotalar joint.
- The shaver needs to glide along the shaft, as the mosquito clamp did, until it reaches the bone; then, it is tilted $45^{\circ}$ in the plantar direction, thereby removing the fatty tissue overlying the joint capsule.

- Remove the thin subtalar joint capsule so that the posterior compartment of the subtalar joint can be visualized.

- The posterior tibiofibular and posterior talofibular ligaments may be recognized. The latter may be removed completely in order to increase visualization of the subtalar joint.

- Always direct the opening of the shaver to the bone, going from posterolateral to posteromedial.

- Find the flexor hallucis longus tendon as it is an important landmark for the more deeply located neurovascular bundle.

- Remove the os trigonum and osseous avulsions in the posterior compartment and reduce the size of the posterolateral process of the talus as necessary. Perform a release of the flexor hallucis longus tendon by cutting its retinaculum.

- A total synovectomy and/or capsulectomy of the posterior aspect of the subtalar joint can be performed. However, try to retain the intermalleolar ligament as well as the fibers of the posterior tibiofibular ligament, serving as a kind of labrum to the joint, in order to avoid debris going into the tibiotalar joint while debriding the subtalar joint.

\section{Step 6: Debridement and Microfracturing of the Posterior Facet of the Subtalar Joint (Fig. 5, Video 6)}

Sometimes an accessory portal, just anterior to the tip of the lateral malleolus, may be needed to achieve a distraction of the anterior part of the subtalar posterior facet.

- Once all of the landmarks are clearly visible, proceed to the debridement of the posterior subtalar articular surface.

- Use curets as well as the full-radius 5-mm shaver for the debridement going from back to front until subchondral bleeding bone is reached. Perform microfracturing of the subchondral bone.

- The interosseous talocalcaneal ligament in the sinus tarsi is the end point to reach, thereby preserving one of the main arteries for talar body vascularization.

- The anteromedial aspect of the articular surface of the posterior subtalar facet-in general, the part where a talocalcaneal coalition may be found-is the most difficult part to reach, very 
often necessitating distraction of the joint through an additional anterolateral portal.

- Make the additional anterolateral portal just in front of the lateral malleolus with direct access to the sinus tarsi. Push the blunt trocar through this portal, just in the back of the sinus tarsi in the anterolateral part of the posterior facet, to perform the distraction.

- The middle and anterior subtalar articular surfaces are not approached. The access to the middle facet from posterior is technically more demanding and quite deep (the instruments are too close to each other when working so deeply), which complicates easy debridement. We always try to take away as much as possible of this middle facet. However, we do not entirely agree with other authors ${ }^{13,14}$ who have stated that compression of the posterior facet is necessary to obtain fusion of the subtalar joint in this arthroscopic procedure. Likewise, an arthroscopic arthrodesis of the ankle does not require contact between surfaces because there often is a void of approximately 3 to $5 \mathrm{~mm}$ between the tibia and the talus. Moreover, the literature is not clear whether the valgus position in a subtalar coalition should be corrected or whether the subtalar joint may be fixed in situ. If it is left in situ, compression does not seem possible in our opinion.

- In general, no invasive traction device is necessary.

\section{Step 7: Use of Bone Graft (Video 6)}

To perform this concomitant step, which generally is not necessary, use an arthroscopic forceps to insert a 5 by 20-mm autograft or allograft in the subtalar joint.

- Use a 6-mm percutaneous trocar to harvest iliac cancellous bone autograft, or use bone plugs from a cancellous bone allograft.

- The dimensions of the graft should be approximately $5 \mathrm{~mm}$ in diameter by 15 to $20 \mathrm{~mm}$ in length.

- Place the graft by using arthroscopic grasping forceps.

- Slightly impact the graft.

\section{Step 8: Fixation of the Subtalar Joint} Using Two Cannulated Screws and Fluoroscopy (Video 7)

Use two cannulated screws with a 7.3-mm diameter; we prefer stabilization screws to compression screws.

- Make the incision dorsoplantar and centered over the heel (Fig. 6).
- Use fluoroscopy to assist the positioning of the Kirschner wires.

- Placement should be from the posterior tuberosity of the calcaneus to the talar body (Fig. 7).

- Once the Kirschner wires are in place, check the free mobility of the tibiotalar joint, which is proof of the nonarticular positioning of the Kirschner wires.

- Once the positioning of the Kirschner wires is validated, perform skin closure before drilling the screws. Skin closure is necessary to try to maintain the reaming material in the debrided area.

- Position the screws and use fluoroscopic control to verify their position.

- The approaches are not closed in various layers. Only the skin is closed with separate stitches of nylon suture, and those may be removed after two to three weeks. No drains are used because the main advantage of the technique is to keep the growth factors in situ. Place a wet compress with a semicompressive bandage and plaster of Paris (Video 8)

\section{Step 9: Additional Surgical Procedures}

Always clean out the posterior compartment of the ankle during the PASTA procedure, as is done during surgical treatment for posterior impingement syndrome, and note that both posterior impingement syndrome and subtalar degenerative changes may thereby be easily addressed through this procedure.

- If necessary, perform posterior arthroscopic tibiotalocalcaneal arthrodesis, lengthening of the Achilles tendon, debridement, and/or microfracturing of an osteochondral lesion of the posterior talar dome or tibial plafond. Perform debridement and microfracturing of osteochondral lesions of the posterior $50 \%$ of the tibial plafond or talar dome (Figs. 8-A and 8-B).

- During the PASTA procedure, one may easily access the tibiotalar joint and perform the classic debridement and microfracturing technique for osteochondral lesions in the posterior part of the tibiotalar joint (the posterior $50 \%$ of the tibial plafond or talar dome) (Figs. 8-A and 8-B).

\section{Step 10: Postoperative Care (Video 9)}

A short posterior leg splint is worn for three to ten days, followed by another non-weight-bearing cast for another three weeks.

- Day 1 through day 10: A short posterior leg splint

- Week 2 through week 4: A non-weight-bearing cast 
- Week 5 through week 8: A removable boot at night and while walking. However, the patient is asked during the day to perform flexion and extension exercises of the ankle regularly without putting weight on the operated side; rotatory movements are discouraged.

- After week 8: Patients are allowed to apply weight according to swelling and pain at the site of the operation, first with the help of crutches and/or a removable boot, and later with normal shoes.

\section{Results}

Since the appearance of the original article, a total of forty-one unilateral subtalar joints in twenty-five men and sixteen women, seen between May 2007 and December 2012, with isolated subtalar arthritis or talocalcaneal coalition without any other major hindfoot arthritis were treated with posterior arthroscopic subtalar arthrodesis (Videos 10 and 11), and all subtalar joints except for two were considered radiographically fused at the first attempt after an average of 6.7 weeks (range, six to ten weeks) ${ }^{4}$. Primarily, bone graft was used in the first eight patients. There was no difference in fusion success rate and fusion time between patients with or without bone-grafting. Preoperative hindfoot alignment was preserved in all patients. The mean American Orthopaedic Foot \& Ankle Society (AOFAS) hindfoot score improved from 49 points (range, 22 to 67 points) to 81 points (range, 60 to 91 points) at the time of the latest follow-up (mean, twenty-two months). The failure rate in the present series was $12 \%$. The two subtalar joints that had a nonunion achieved union after a second operative attempt with open surgery and bone-grafting. Three patients operatively treated for talocalcaneal coalition presented postoperatively with a lateral submalleolar impingement. Two of them were successfully treated with orthopaedic insoles, and the third required a resection of the calcaneal lateral edge. Because of the minimally invasive method, the rehabilitation protocol was adapted by starting mobilization exercises four weeks earlier than for the open procedure. Patients were satisfied with the cosmetic outcome of the procedure. At the latest review, all patients were free of symptoms.

\section{What to Watch For}

\section{Indications}

- Primary and posttraumatic osteoarthritis.

- Osteochondral lesions in the central part of the subtalar posterior facet.

- Symptomatic talocalcaneal synostosis or synchondrosis.

- Inflammatory systemic diseases with osteoarthritic changes of the subtalar joint.
- Tibialis posterior tendinopathy.

- Subtalar joint arthrosis after total ankle replacement or tibiotalar arthrodesis.

- Revision arthrodesis.

\section{Contraindications}

- As shown in the decision algorithm (Fig. 1), certain types of degenerative changes to the subtalar joints are better addressed through open procedures:

- When there is important bone loss in the posterior subtalar joint, it is often necessary to lift up the talar dome to remove the talar neck of the anterior tibial plafond and thereby decrease the anterior impingement syndrome. This may only be achieved through the open procedure as the middle and anterior facets are not addressed during the PASTA procedure.

- When there is lateral wall impingement, the posterior as well as the submalleolar part of the calcaneus, but not the front portion, may be removed. There also is a risk of damaging the peroneal tendons.

- In patients with malrotation of the talus on the calcaneus or dislocation of the Chopart joint, open subtalar arthrodesis with derotational osteotomies or additional extraarticular osteotomies may be mandatory. An alternative, if the rotational problem is quite distal, is a triple arthrodesis.

- The presence of active septic arthritis demands a two-stage procedure with debridement and lavage of the infected joint, followed by a reconstruction, in general, through the classic open approach.

- For reasons related to anesthesia, certain patients, such as those who are very obese, will not be eligible for the prone position.

\section{Pitfalls \& Challenges}

- During the entire procedure, keep the flexor hallucis longus tendon as the most medial reference point. Often, especially in patients with talocalcaneal coalition, the debridement at the posteromedial or sustentaculum tali level will be the most laborious. The posterior tibial nerve and the flexor hallucis longus tendon are the critical structures that are at greatest risk during this procedure.

- There are a lot of anatomical variants in the orientation of the posterior facet of the subtalar joint, with the flat surface being easier to debride than the more convex surface. 
- Avoid resection of the intermalleolar ligament as it will function as a barrier during debridement, preventing debris extravasation into the anterior ankle compartment. Once the debridement is done, evaluate the cartilage in the tibiotalar joint as needed.

- As the interosseous ligament is preserved, it functions as a pivot point. In patients with a subtalar fusion on the left side, use compression screws to induce a superposition of the talus on the calcaneus and thereby provoke a triplanar deformity. Figure 9 demonstrates this superposition with varus of the hindfoot as a consequence.

- A talar body fracture is often ignored, leading to delayed diagnosis with degenerative changes to the tibiotalar and subtalar joints, talar fracture nonunion, and/or development of a cystic osteochondral lesion. A challenge may be to treat the nonunion through the same approach, with additional portals if necessary, while performing the PASTA procedure (Fig. 10).

- Even in a well-aligned subtalar joint with degenerative changes underneath a tibiotalar arthrodesis, we think that the PASTA procedure should be followed by a more severe postoperative regimen, with a longer duration of cast immobilization and a prolonged period of non-weightbearing, as patients with these circumstances were the only ones in whom the procedure failed to obtain fusion.

- Removal of osteosynthesis devices used for a previous calcaneal fracture is not always necessary if the new screws have been passed into the posterior talar facet without having a conflict with the previously implanted material.

\section{Clinical Comments}

- Bone-grafting of the debrided posterior facet does not seem necessary, despite all of the good results at the beginning. The remaining $80.5 \%$ of the patients had not received any graft material, and fusion occurred in all patients, except for those who had the PASTA procedure underneath a tibiotalar fusion.
- A tibiotalar fusion is considered a contraindication as it is the only clinical setting in which this PASTA procedure did not lead to fusion and needed revision with an open procedure and bone-grafting. We believe that the tibiotalar fusion leads to too many constraints at the middle and anterior facets of the subtalar joint, as well as at the Chopart joint, and that the limited fusion of the posterior facet is insufficient (Fig. 11).

- In all other patients, this union of only the subtalar posterior facet seemed sufficient at the time of medium and long-term follow-up.

- During surgery, try to maintain as much of the posterolateral talar tubercle as possible, especially in the plantar direction, to increase the surface of contact for union. However, at the same time, diminish its dorsal contour in order to treat the posterior impingement syndrome that may be associated with it.

- The posterior arthroscopic approach, compared with the sinus tarsi approach, better preserves the talar vascular supply. The two main arteries for talar body vascularization are the artery of the tarsal canal (originating from the posterior tibial artery) and the artery of the sinus tarsi (originating from the dorsalis pedis and peroneal arteries), anastomosing in the tarsal canal. Thus, the posterior approach damages only the few posterior nutrient arteries.

- The prone position seems ideal for an easy positioning of the screws.

- In patients with subtalar synchondrosis or synostosis, this technique offers a good alternative to open procedures. The question of whether the valgus position needs to be corrected in order to avoid a lateral submalleolar impingement or whether an arthrodesis in situ should be performed continues to be debated in the literature ${ }^{15}$.

- Compared with open procedures, the PASTA procedure seems to be a less invasive and more secure procedure to address subtalar lesions without associated hindfoot or forefoot deformities. 
Bernhard Devos Bevernage, MD

Laurent Goubau, MD

Paul-André Deleu, MSc

Vincent Gombault, MD

Pierre Maldague, MD

Thibaut Leemrijse, MD

Foot \& Ankle Institute, Brussels, Belgium

E-mail address for B. Devos Bevernage: bdevosb@ hotmail.com

E-mail address for L. Goubau: laurent.goubau@gmail.com

E-mail address for P.-A. Deleu: pa.deleu@gmail.com

E-mail address for V. Gombault: gombault@footandankleinstitute.be

E-mail address for P. Maldague: drpierremaldague@gmail.com

E-mail address for T. Leemrijse: thibaut.leemrijse@gmail.com

Disclosure: None of the authors received payments or services, either directly or indirectly (i.e., via his or her institution), from a third party in support of any aspect of this work. None of the authors, or their institution(s), have had any financial relationship, in the thirtysix months prior to submission of this work, with any entity in the biomedical arena that could be perceived to influence or have the potential to influence what is written in this work. Also, no author has had any other relationships, or has engaged in any other activities, that could be perceived to influence or have the potential to influence what is written in this work. The complete Disclosures of Potential Conflicts of Interest submitted by authors are always provided with the online version of the article.

\section{References}

1. Scranton PE Jr. Comparison of open isolated subtalar arthrodesis with autogenous bone graft versus outpatient arthroscopic subtalar arthrodesis using injectable bone morphogenic protein-enhanced graft. Foot Ankle Int. 1999 Mar;20(3):162-5.

2. Glanzmann MC, Sanhueza-Hernandez R. Arthroscopic subtalar arthrodesis for symptomatic osteoarthritis of the hindfoot: a prospective study of 41 cases. Foot Ankle Int. 2007 Jan;28(1):2-7.

3. Tasto JP. Arthroscopy of the subtalar joint and arthroscopic subtalar arthrodesis. Instr Course Lect. 2006;55:555-64.

4. Albert A, Deleu PA, Leemrijse T, Maldague P, Devos Bevernage B. Posterior arthroscopic subtalar arthrodesis: ten cases at oneyear follow-up. Orthop Traumatol Surg Res. 2011 Jun;97(4):401-5. Epub 2011 May 12.

5. Amendola A, Lee KB, Saltzman CL, Suh JS. Technique and early experience with posterior arthroscopic subtalar arthrodesis. Foot Ankle Int. 2007 Mar;28(3):298-302.

6. Carro LP, Golanó P, Vega J. Arthroscopic subtalar arthrodesis: the posterior approach in the prone position. Arthroscopy. 2007 Apr;23(4):445.e1-4. Epub 2007 Feb 20.

7. Beimers L, de Leeuw PA, van Dijk CN. A 3-portal approach for arthroscopic subtalar arthrodesis. Knee Surg Sports Traumatol Arthrosc. 2009 Jul;17(7):830-4. Epub 2009 Apr 17.

8. El Shazly O, Nassar W, El Badrawy A. Arthroscopic subtalar fusion for post-traumatic subtalar arthritis. Arthroscopy. 2009 Jul;25(7):783-7. Epub 2009 Mar 17.

9. Lee KB, Park CH, Seon JK, Kim MS. Arthroscopic subtalar arthrodesis using a posterior 2-portal approach in the prone position. Arthroscopy. 2010 Feb;26(2):230-8. Epub 2009 Dec 21.

10. Saltzman CL, el-Khoury GY. The hindfoot alignment view. Foot Ankle Int. 1995 Sep;16(9):572-6.

11. Méary P, Filip G, Aubriot JH, Tomeno B. [Etude fonctionnelle de la double arthrodèse du pied]. Rev Chir Orthop Repar Appar Mot. 1977 Apr;63(4):345-59. French.

12. van Dijk CN, Scholten PE, Krips R. A 2-portal endoscopic approach for diagnosis and treatment of posterior ankle pathology. Arthroscopy. $2000 \mathrm{Nov} ; 16(8): 871-6$.

13. Hintermann B, Valderrabano V, Nigg B. Influence of screw type on obtained contact area and contact force in a cadaveric subtalar arthrodesis model. Foot Ankle Int. 2002 Nov; 23(11):986-91.

14. Chuckpaiwong B, Easley ME, Glisson RR. Screw placement in subtalar arthrodesis: a biomechanical study. Foot Ankle Int. 2009 Feb; 30(2):133-41.

15. Thorpe SW, Wukich DK. Tarsal coalitions in the adult population: does treatment differ from the adolescent? Foot Ankle Clin. 2012 Jun; 17(2):195-204. 


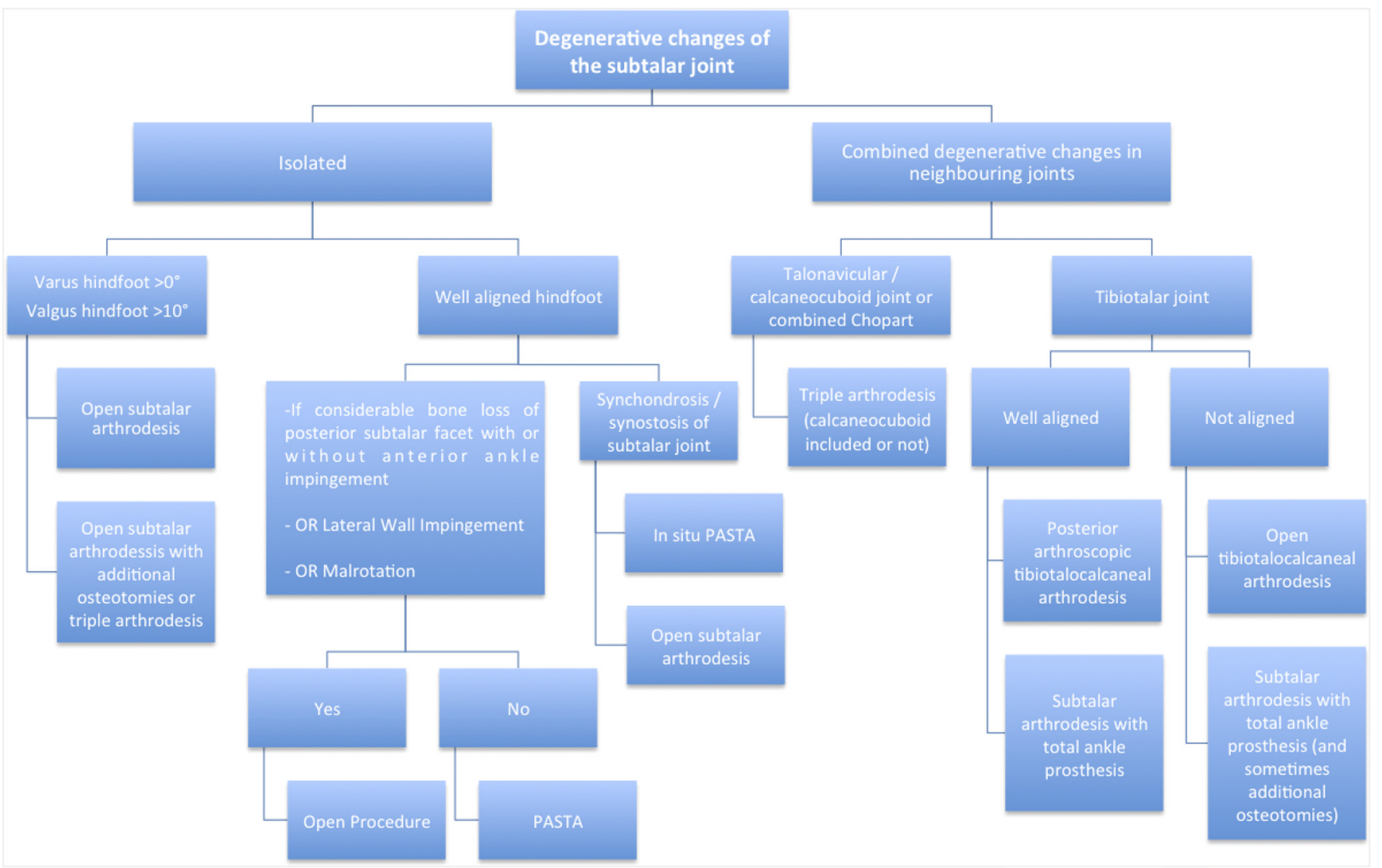

Fig. 1

Decision algorithm for the treatment of patients with subtalar degenerative joint disease. PASTA = posterior arthroscopic subtalar arthrodesis.

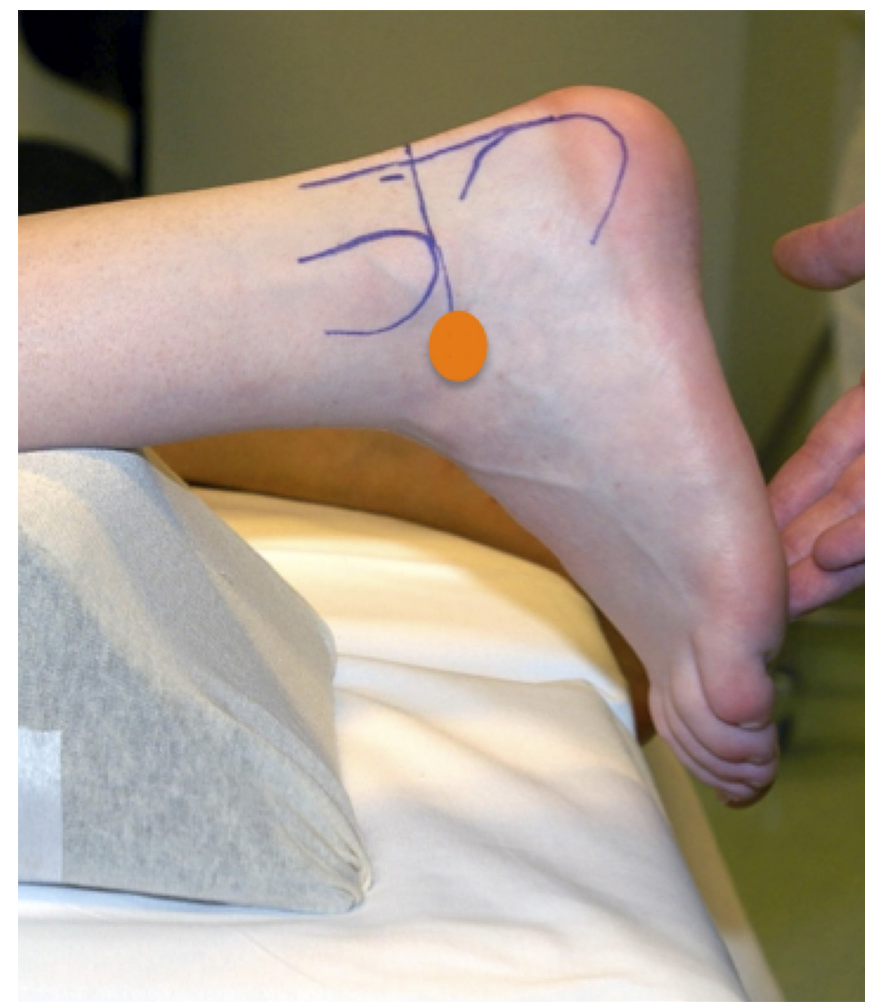

Fig. 2

First mark the tip of the lateral malleolus and the lateral border of the Achilles tendon. Draw a line between those two landmarks while holding the ankle in neutral position. Make a vertical stab incision on the lateral border of the Achilles tendon, proximal to this line. The orange oval indicates the location of the anterolateral portal, which helps in the distraction of the anterior part of the posterior subtalar facet. 


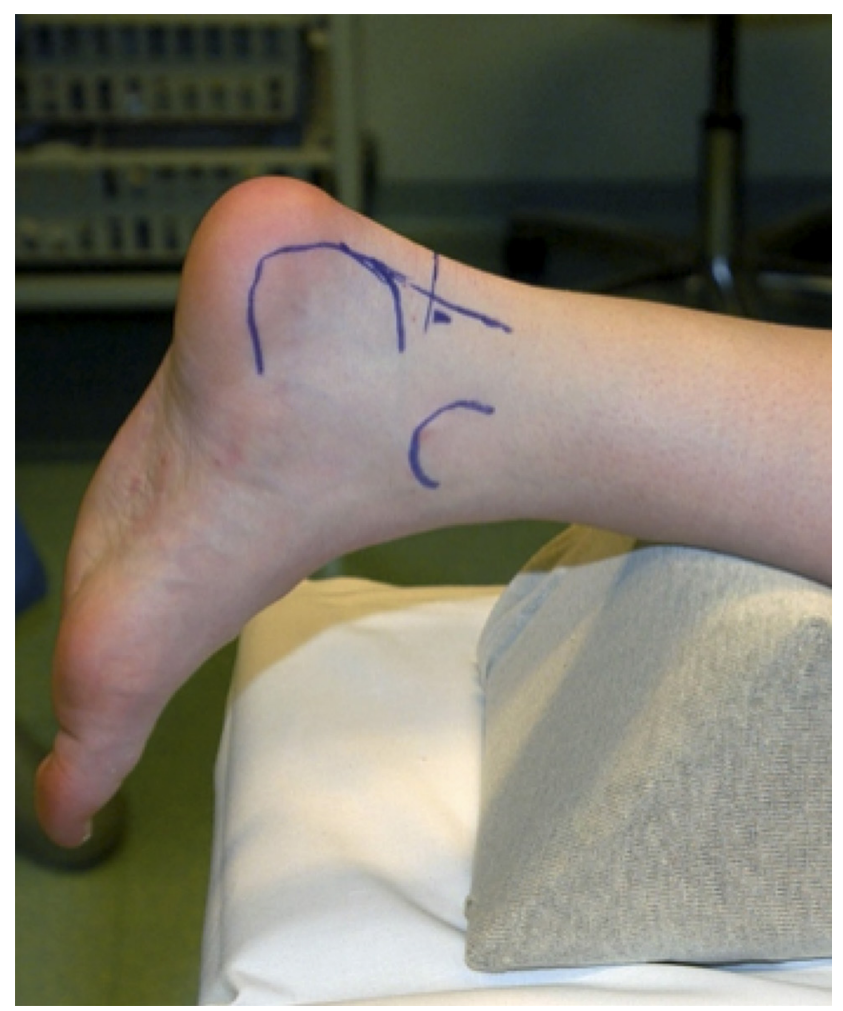

Fig. 3

The posteromedial portal is made just medial to the Achilles tendon at the same level as the posterolateral portal in the coronal plane.

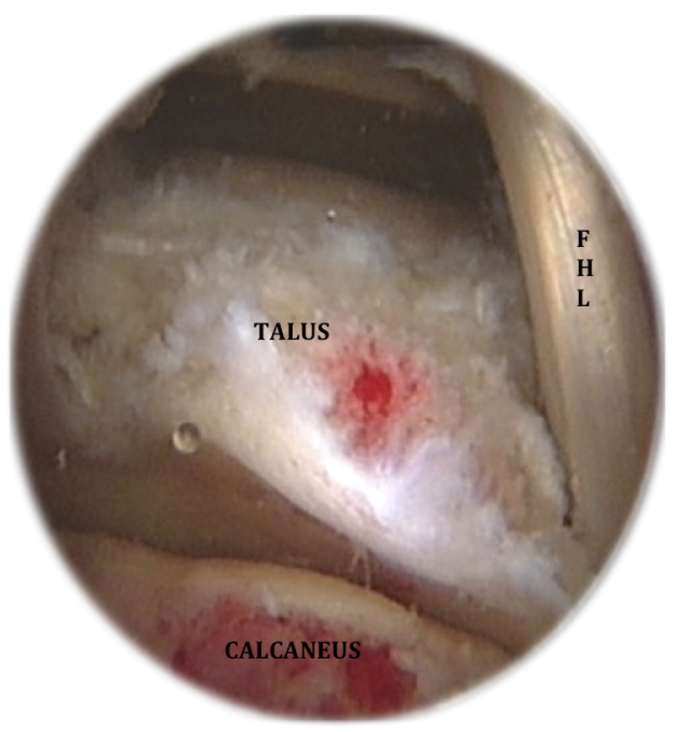

Fig. 4

Arthroscopic view of both the tibiotalar and subtalar joints after removal of the fatty tissue, part of the posterior joint capsule, together with the intermalleolar ligament, as well as the hypertrophic posterolateral tubercle of the talus. $\mathrm{FHL}=$ flexor hallucis longus 


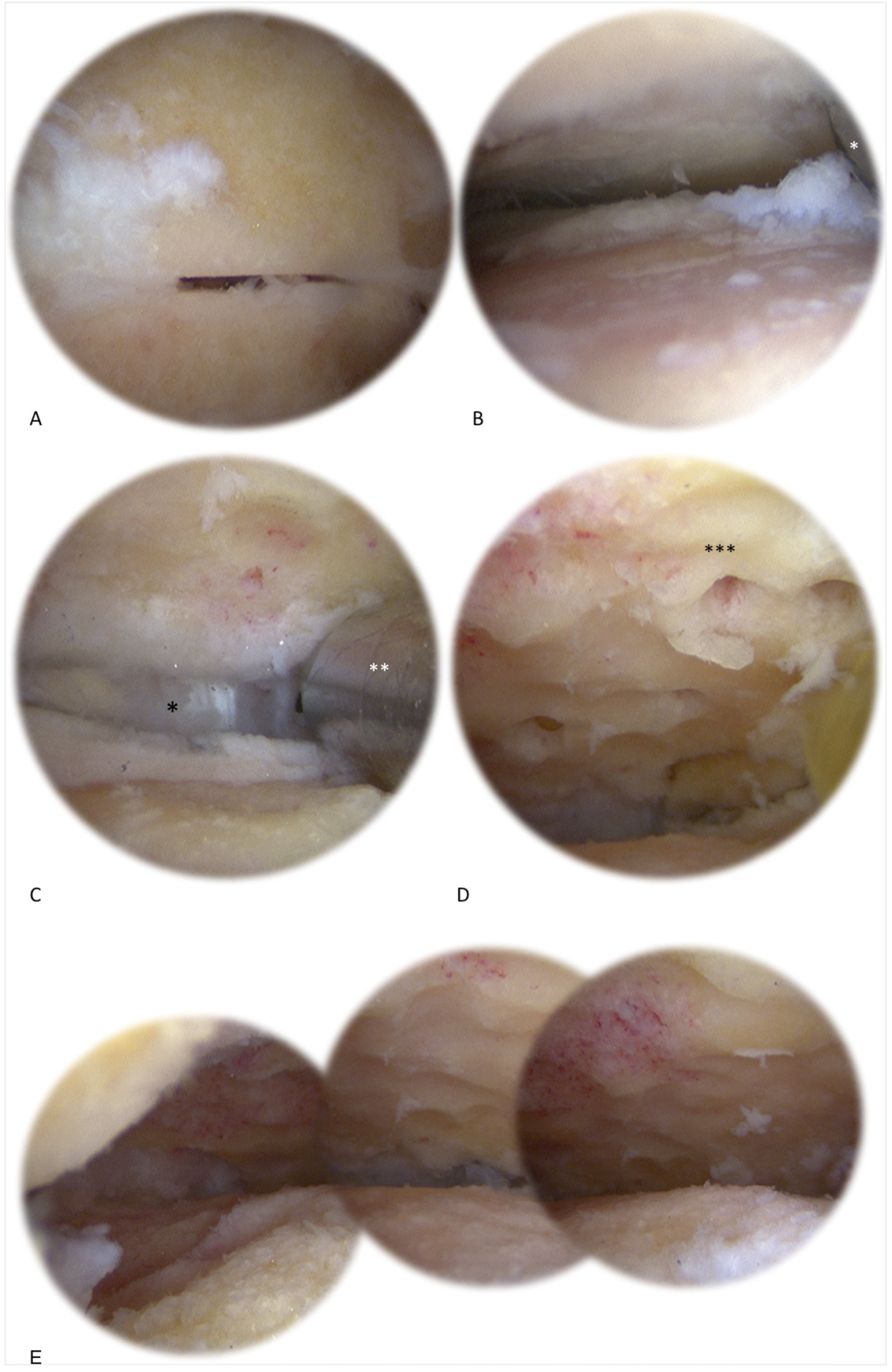

Fig. 5

Figs. 5-A through 5-E Arthroscopic views of the posterior aspect of the subtalar joint. Fig. 5-A The tightness of the posterior subtalar facet, which sometimes needs distraction with a blunt trocar introduced into the subtalar joint from the sinus tarsi through an additional anterolateral portal, is evident. Fig. 5-B Advanced chondropathy of the subtalar posterior facet is visible on distraction (white asterisk) at the anterolateral part of the subtalar joint. Fig. 5-C Arthroscopic view of the debridement of the posterior subtalar facet performed from posterior to anterior, until the interosseous talocalcaneal ligament is reached. The one black asterisk indicates the interosseous talocalcaneal ligament, and the two white asterisks indicate the full-radius shaver. Fig. 5-D At the end of the debridement, microfracturing is performed. The three black asterisks indicate microfracturing after debridement. Fig. 5-E Arthroscopic overview of the debridement of the posterior subtalar facet. 


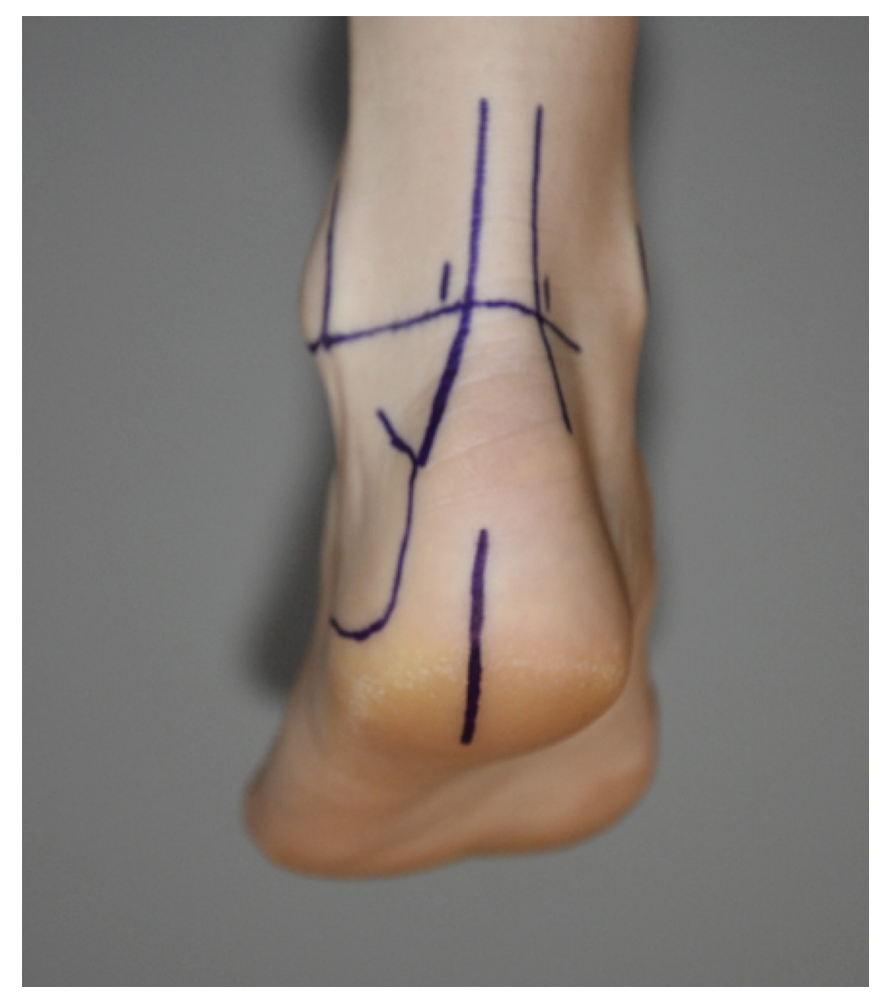

Fig. 6

An incision is made in the center of the heel region to allow placement of the cannulated screws from the posterior tuberosity of the calcaneus to the talar body.

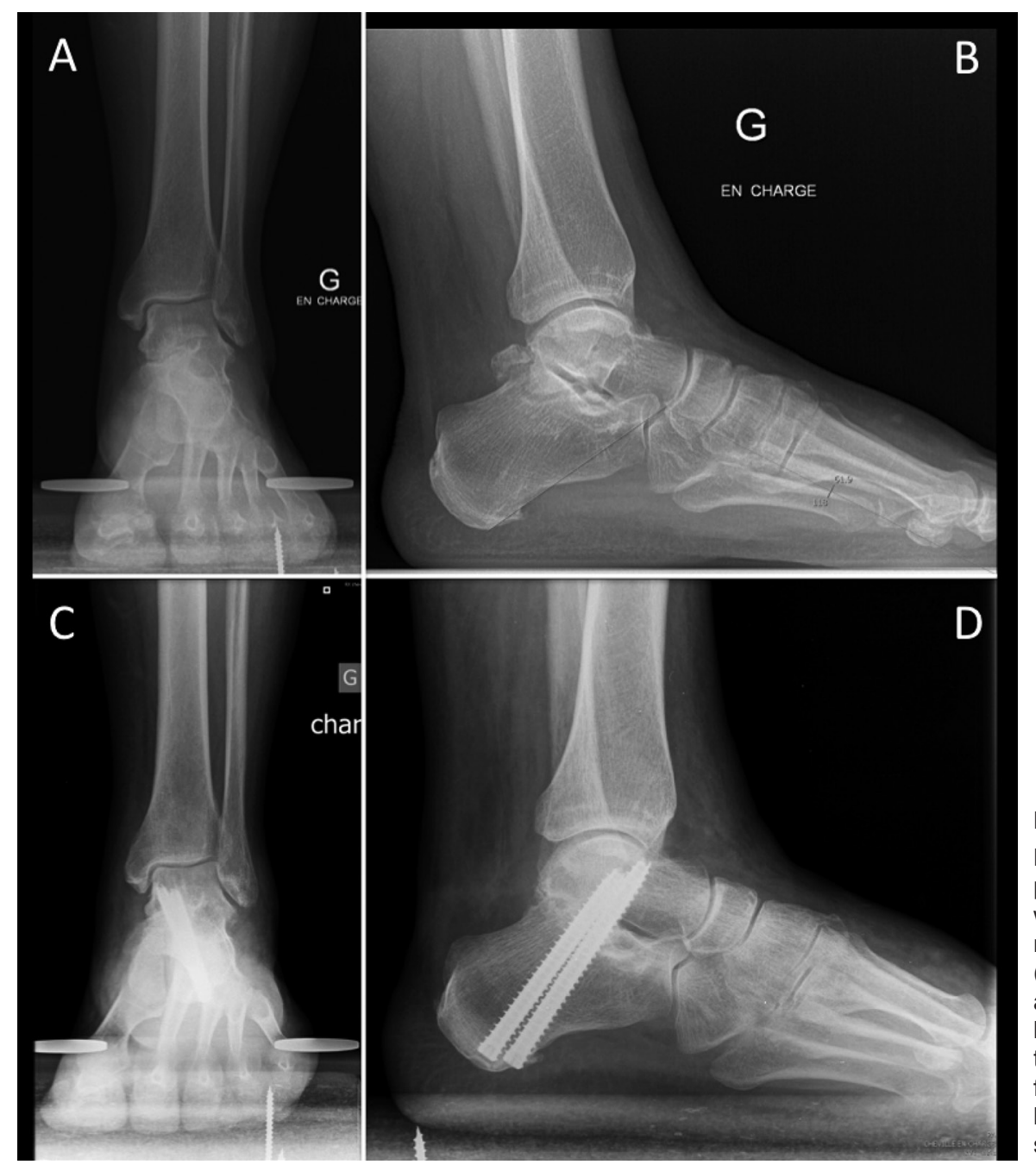

Fig. 7

Figs. 7-A through 7-D The PASTA procedure in a patient who had a degenerative subtalar joint associated with posterior ankle impingement caused by os trigonum. Figs. 7-A and 7-B Preoperative anteroposterior (Fig. 7-A) and lateral (Fig. 7-B) radiographs. Figs. 7-C and 7-D Postoperative anteroposterior (Fig. 7-C) and lateral (Fig. 7-D) radiographs after the subtalar joint arthrodesis with the cannulated stabilization screws placed from the posterior tuberosity of the calcaneus to the talar body. The resection of the os trigonum was done at the same time. 


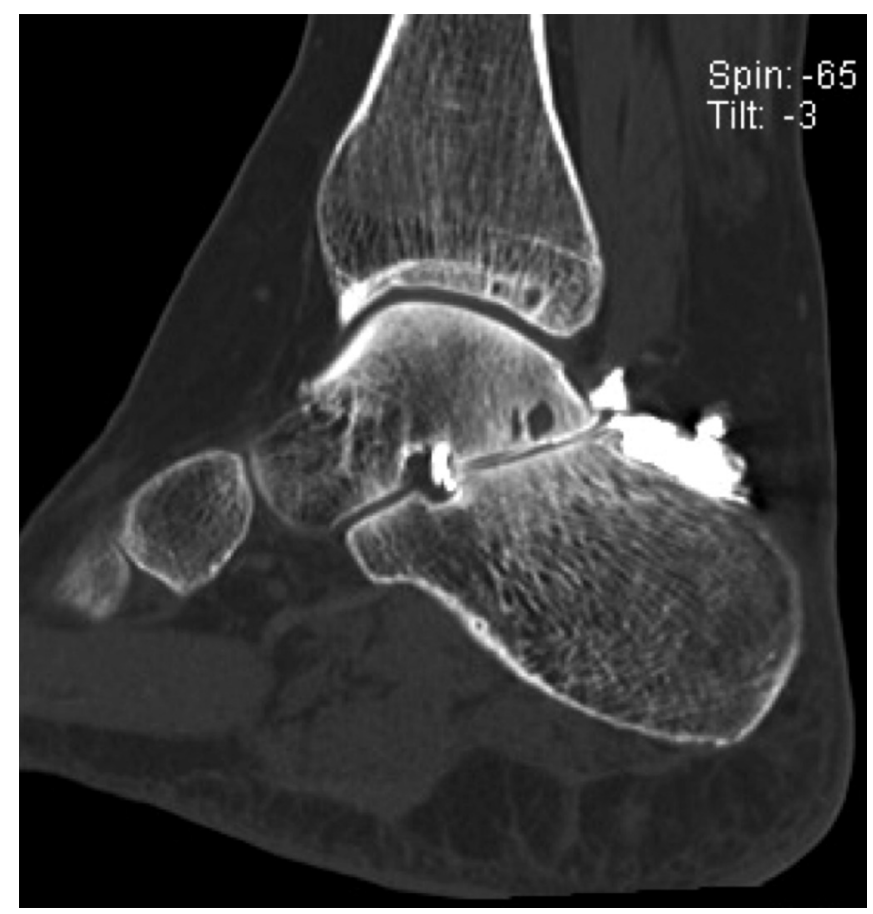

Fig. 8-A

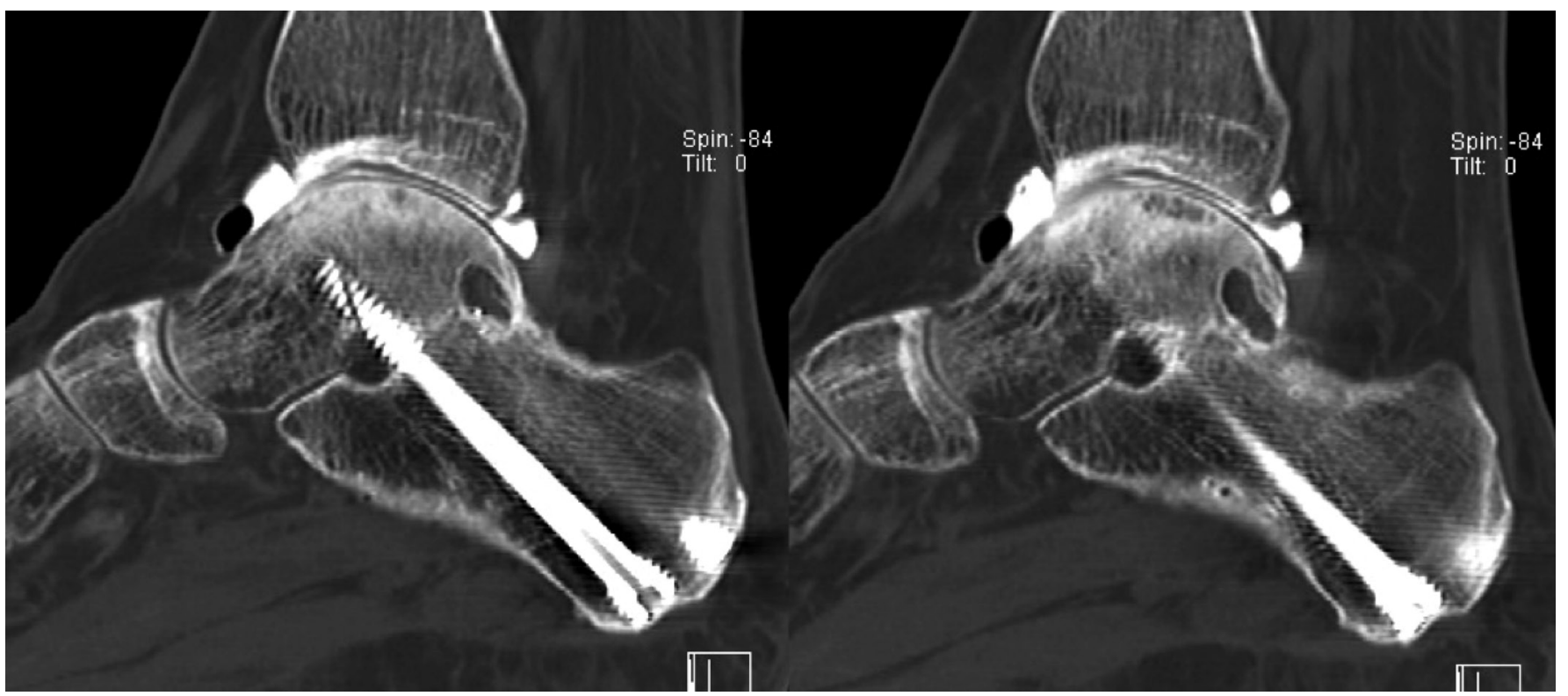

Fig. 8-B

Figs. 8-A and 8-B A patient with clearly degenerative changes in the subtalar joint and an osteochondral lesion of the tibial plafond who was an ideal candidate for the PASTA procedure, in combination with arthroscopic debridement and microfracture of the osteochondral lesion. Fig. 8-A Preoperative CT scan with arthrography (arthro-CT). Fig. 8-B Postoperative arthro-CT scans showing subtalar fusion. The patient continued to have pain but only at the tibiotalar joint because of an associated osteochondral lesion of the talar dome that existed before the subtalar fusion procedure. 

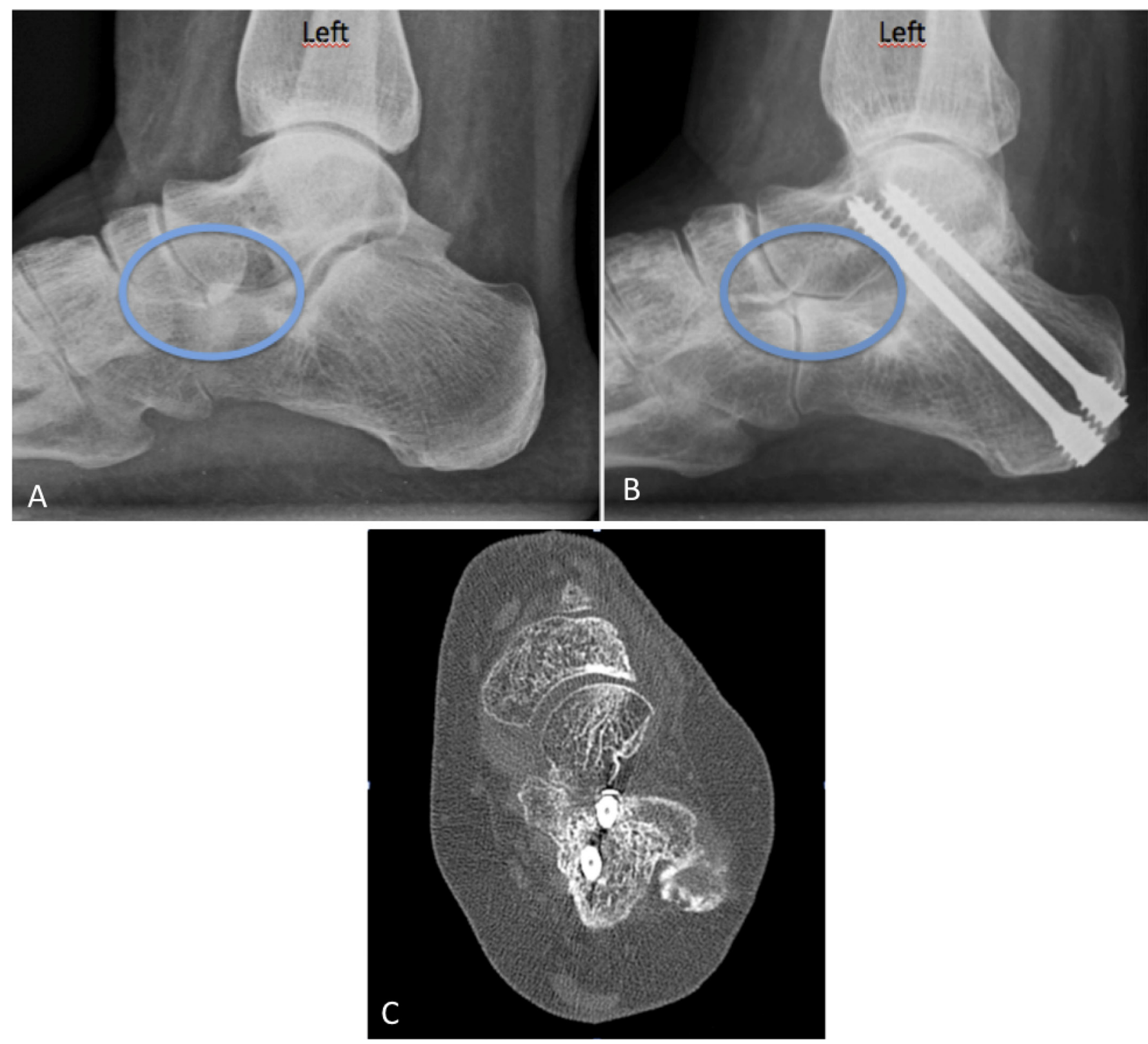

Fig. 9

Figs. 9-A, 9-B, and 9-C A patient with isolated subtalar primary arthritis who was managed with the PASTA procedure with the use of compression screws. Fig. 9-A Preoperative lateral standing radiograph. Fig. 9-B Postoperative lateral standing radiograph. Notice the elevation of the talus onto the calcaneus (blue ovals) because of compression posteriorly and the twist of the talus while the screws were inserted. The patient continued to report pain deep in the Chopart joint, as well as the impression of walking on the lateral side of the foot. Fig. 9-C Axial CT scan showing the talonavicular incongruence. 


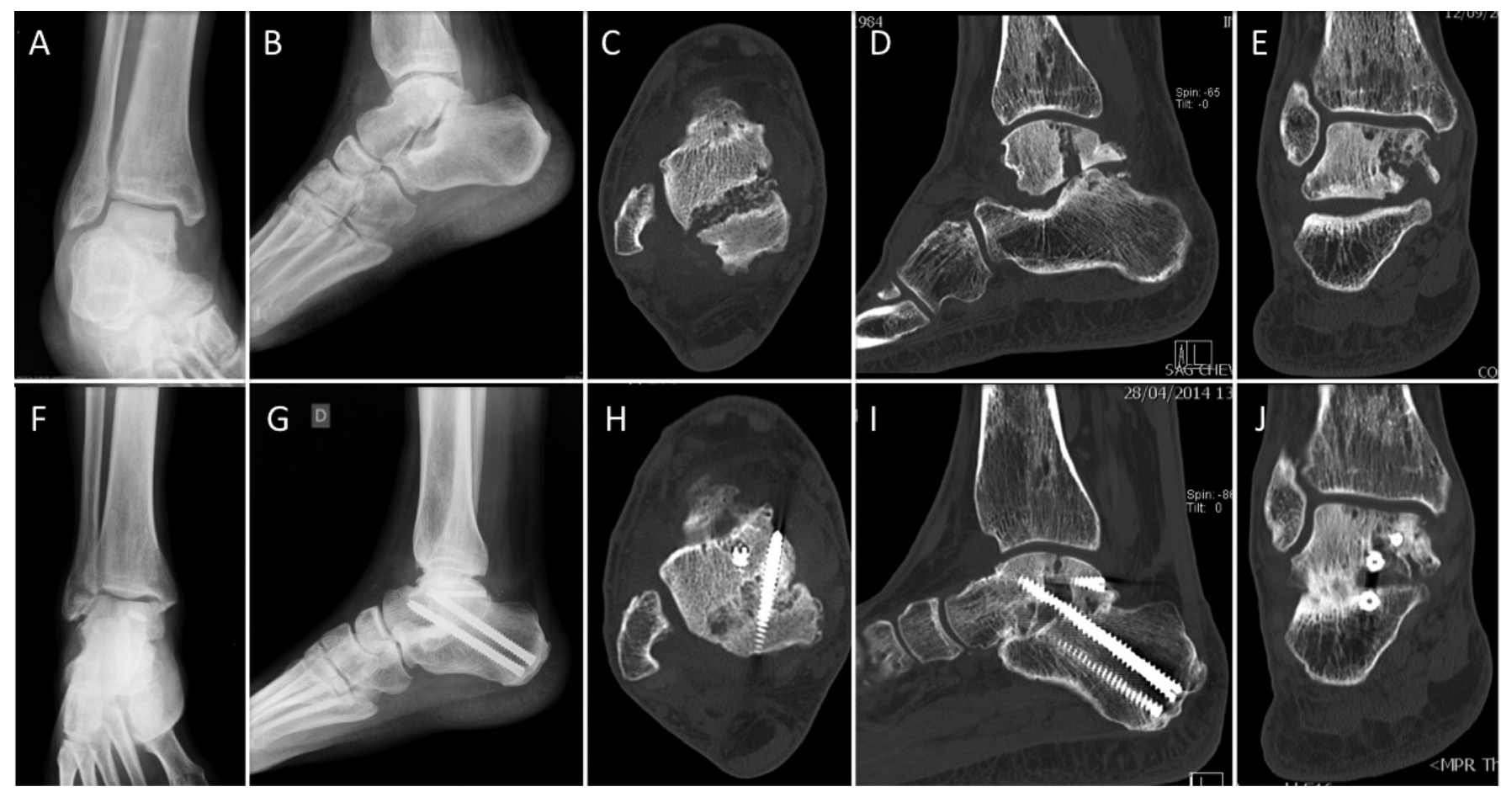

Fig. 10

Figs. 10-A through 10-J A patient with osteoarthritis of a partially dislocated subtalar joint, seen fourteen months after a neglected fracture of the talar body that presented as a nonunion, who was managed with arthroscopic debridement of the nonunion and the posterior facet of the subtalar joint. Figs. 10-A through 10-E Preoperative radiographic images, including anteroposterior and lateral radiographs (Figs. 10-A and 10-B) and axial (Fig. 10-C), lateral (Fig. 10-D), and coronal (Fig. 10-E) CT scans. Figs. 10-F through 10-J Postoperative radiographic images, including anteroposterior and lateral radiographs (Figs. 10-F and 10-G) and axial (Fig. 10-H), lateral (Fig. 10-I), and coronal (Fig. 10-J) CT scans, showing a fusion of the talar body fracture as well as of the subtalar joint.
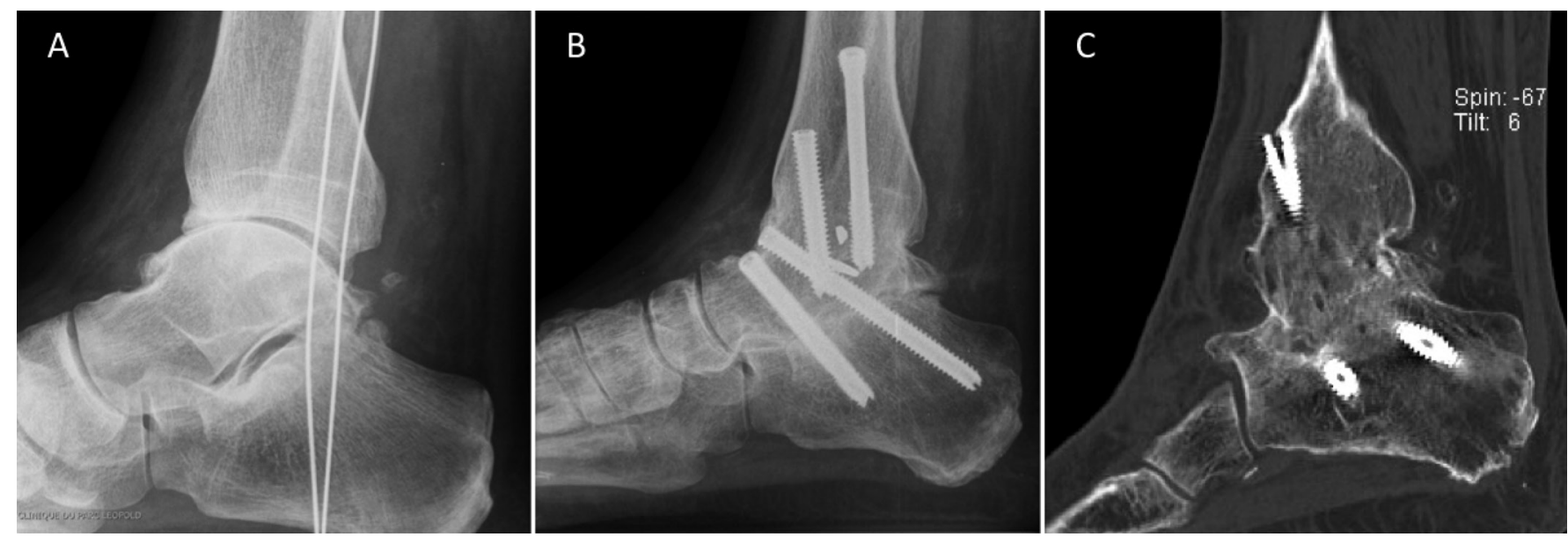

Fig. 11

Figs. 11-A, 11-B, and 11-C A patient with a combined pathology of tibiotalar and subtalar degenerative joint disease, with most of the symptoms found in the tibiotalar joint and no improvement after an infiltration of a corticosteroid derivative under fluoroscopic control in the subtalar joint, who was managed with an arthroscopic ankle arthrodesis. Fig. 11-A Preoperative lateral standing radiograph. Fig. 11-B Lateral radiograph made after a PASTA procedure, performed three years after a tibiotalar fusion because the patient had developed pain in the subtalar joint and was not able to meet functional demands. However, the patient had no pain relief because of a nonunion that was confirmed on a CT scan made at eight months postoperatively. The subtalar fusion was revised through an open approach with the use of bone-grafting. Fig. 11-C Sagittal CT scan, made after the open procedure with bone-grafting underneath the tibiotalar arthroscopic fusion, showing the fusion of the subtalar joint. 